\title{
A novel danshensu/tetramethypyrazine derivative attenuates oxidative stress-induced autophagy injury via the AMPK-mTOR-Ulk1 signaling pathway in cardiomyocytes
}

\author{
CAIPENG XIE $^{1}$, JINGXIONG LUO ${ }^{1}$, HUIHUI HU ${ }^{1}$, LIANG WANG ${ }^{2}$, PEI YU ${ }^{1}$, \\ LIPENG XU ${ }^{1}$, YEWEI SUN ${ }^{1}$, YUQIANG WANG ${ }^{1}$ and LUCHEN SHAN ${ }^{1}$ \\ ${ }^{1}$ Institute of New Drug Research and Guangzhou Key Laboratory of Innovative Chemical Drug Research in \\ Cardio-cerebrovascular Diseases, Jinan University College of Pharmacy, Guangzhou, Guangdong 510000; \\ ${ }^{2}$ Institute of Biomedical and Pharmaceutical Sciences, Guangdong University of Technology, \\ Guangzhou, Guangdong 510006, P.R. China
}

Received March 9, 2020; Accepted October 27, 2020

DOI: $10.3892 /$ etm.2020.9550

\begin{abstract}
Myocardial ischemia/reperfusion injury (MIRI) is an inevitable and unsolved clinical problem in the treatment of ischemic heart diseases. Compound DT-010 is a novel danshensu/tetramethylpyrazine derivative and was examined as a candidate for treating MIRI. In the present study, MTT, lactate dehydrogenase assay and Hoechst staining data indicated that DT-010 attenuated tert-butylhydroperoxide (t-BHP)-induced oxidative damage by increasing cell survival, reducing cell damage and decreasing apoptosis in H9c2 cardiomyocytes. Autophagy was assessed by western blotting for microtubule-associated protein 1A/1B-light chain 3 (LC3-II and LC3-I) expression, acridine orange and monodansylcadaverine staining for autophagosome formation and the monomeric red fluorescent protein-green fluorescent protein-LC3 assay for autophagic flow. t-BHP-induced cell damage was aggravated by the autophagy agonist rapamycin and alleviated by the autophagy blocker hydroxy-chloroquine, suggesting that autophagy was involved in t-BHP-induced
\end{abstract}

Correspondence to: Dr Liang Wang, Institute of Biomedical and Pharmaceutical Sciences, Guangdong University of Technology, 100 Waihuanxi Road, University Town, Panyu, Guangzhou, Guangdong 510006, P.R. China

E-mail: wangliangjnu@163.com

Dr Luchen Shan, Institute of New Drug Research and Guangzhou Key Laboratory of Innovative Chemical Drug Research in Cardio-cerebrovascular Diseases, Jinan University College of Pharmacy, 601 West Huangpu Avenue, Guangzhou, Guangdong 510000, P.R. China

E-mail: ytysxs@126.com

Key words: danshensu/tetramethylpyrazine derivative, cardioprotection, oxidative stress, autophagy, 5'-AMP-activated protein kinase/mTOR/unc-51 like autophagy activating kinase 1 signaling pathway cardiomyocyte injury. DT-010 pretreatment significantly prevented t-BHP-induced cell damage, which was partially but significantly abolished by rapamycin and significantly improved by hydroxy-chloroquine treatment. DT-010 treatment inhibited t-BHP-induced autophagy in H9c2 cells, reduced phosphorylation of 5'-AMP-activated protein kinase (AMPK) and promoted the phosphorylation of mTOR and unc-51 like autophagy activating kinase 1 (Ulk1). To conclude, DT-010 can serve as a potential candidate for myocardial ischemia-reperfusion injury therapy. The cardioprotective effects of DT-010 could be partially attributed to its inhibition of autophagy via the AMPK-mTOR-Ulk1 signaling pathway.

\section{Introduction}

Ischemic heart disease is a serious condition due to its high morbidity and mortality, which is responsible for $\sim 40 \%$ deaths associated with cardiovascular diseases worldwide (1). At present, timely restoration of blood flow is a generally accepted strategy that is effective for improving ischemic heart injury $(2,3)$. However, reperfusion may trigger several adverse responses, including calcium overload, oxidative-nitrosylation stress, endoplasmic reticulum stress, mitochondrial dysfunction and inflammation, leading to cell death and heart dysfunction $(2,3)$. Therefore, myocardial ischemia/reperfusion injury is an essential clinical problem worldwide that has attracted increasing attention from clinicians. An increasing number of studies have focused on strategies for alleviating this condition.

A number of studies have previously demonstrated that cardiomyocyte autophagy is increased in both acute ischemia-reperfusion (4-6) and chronic ischemia models $(7,8)$. Autophagy is increasingly recognized as a potential target for the treatment of myocardial ischemia-reperfusion $(9,10)$ and is generally activated by oxidative stress, hypoxia and starvation, which is physiologically a cell defense and adaption mechanism to promote cell survival (11). However, excessive and uncontrolled autophagy can result in cell death (12). Therefore, maintaining autophagy at a reasonable level is essential for 
determining cell fate. Tert-butylhydroperoxide (t-BHP) is an organic peroxide compound that is used widely as an alternative to $\mathrm{H}_{2} \mathrm{O}_{2}$ in oxidative stress studies to mimic cellular oxidative damage (13-15). In addition to the increased production of reactive oxygen species (ROS), t-BHP-induced cell death can also result from the activation of autophagy $(16,17)$. Therefore, inhibition of autophagy may protect against t-BHP-induced H9c2 cardiomyocyte death (15).

Traditional Chinese medicine provide an important platform for the discovery of novel therapeutic agents. Danshen and Chuanxiong are two common traditional Chinese medicines that have been used widely for myocardial ischemia therapy $(18,19)$. Danshensu (DSS) and tetramethypyrazin (TMP) are the major active components present within Danshen and Chuanxiong, which exert various pharmacological properties on the cardiovascular system $(18,19)$. In particular, compound DT-010 (Fig. 1) is a patented compound derived from DSS and TMP that was previously synthesized (20). A previous study demonstrated the protective activities of DT-010 against myocardial ischemia and reperfusion injury in a rat model, where DT-010 treatment reduced the infarct size (20). The cardioprotective effect of DT-010 is more potent compared with that of DSS and TMP (21). This previous study also revealed that DT-010 markedly increased cell viability and suppressed cell apoptosis in t-BHP-treated H9c2 cells via activation of the peroxisome proliferator-activated receptor $\gamma$ coactivator (PGC)-1/nuclear factor erythroid 2-related factor 2 (Nrf2)/transcription factor A and the mitochondrial/heme oxygenase-1 (HO-1) signaling pathway (20). In addition, DT-010 has also been reported to prevent doxorubicin-induced cardiotoxicity by inhibiting apoptosis, ROS generation and autophagosome formation (21).

It was speculated that autophagy may also be involved in the protective effects of DT-010 against myocardial ischemia-reperfusion injury. Therefore, the present study established a t-BHP-induced oxidative injured cell model using H9c2 cardiomyocytes and evaluated the effect of DT-010 on autophagy and its underlying regulatory signaling mechanism.

\section{Materials and methods}

Materials. t-BHP, acridine orange (AO), monodansylcadaverine (MDC), MTT, rapamycin and hydroxy-chloroquine were obtained from Sigma-Aldrich, Merck KGaA. Hoechst 33342 was obtained from Molecular Probes, Thermo Fisher Scientific, Inc. The primary antibody against microtubuleassociated protein 1A/1B-light chain 3 (LC3; cat. no. L8918) was purchased from Sigma-Aldrich, Merck KGaA. Primary antibodies against $\beta$-actin (cat. no. 4967), p62 (cat. no. 8025), mTOR (cat. no. 2983), 5'-AMP-activated protein kinase (AMPK; cat. no. 5831), unc-51 like autophagy activating kinase 1 (Ulk1; cat.no.8054),phosphorylated(p)-mTOR(cat.no.5536),p-AMPK (cat. no. 2535) and p-Ulk1 (cat. no. 14202) were obtained from Cell Signaling Technology, Inc. All primary antibodies were rabbit anti-rat antibodies (dilution, 1:1,000). Anti-rabbit IgG, horseradish peroxidase-conjugated antibody (cat. no. 7074) was purchased from Cell Signaling Technology, Inc. Monomeric red fluorescent protein (mRFP)-green fluorescent protein (GFP)-LC3 adenovirus (cat. no. HB-AP21000001; https: //www. hanbio.net/cn/services/item/7) was purchased from Hanbio
Biotechnology Co., Ltd. The lactate dehydrogenase (LDH) kit (cat. no. C0016) was obtained from Beyotime Institute of Biotechnology. DMEM and FBS were obtained from Thermo Fisher Scientific, Inc. The purity of DT-010 was $>95 \%$ (22).

Cell culture. The rat myocyte cell line H9C2 was obtained from The Cell Bank of Type Culture Collection of Chinese Academy of Sciences. H9c2 cardiomyocytes were maintained in DMEM containing 10\% FBS. A humidified atmosphere containing $5 \% \mathrm{CO}_{2}$ at $37^{\circ} \mathrm{C}$ was required for cell growth and cell treatment. Cells were passaged or treated when they reached $\sim 80 \%$ confluence.

MTT and LDH measurement. Cell survival was detected using MTT and LDH kits. To assess cytotoxicity, cells were seeded into 96-well plates at a density of $1 \times 10^{4}$ cells/well and cultured at $37^{\circ} \mathrm{C}$. After $24 \mathrm{~h}, \mathrm{DT}-010$ was added alone for $1 \mathrm{~h}$ at $37^{\circ} \mathrm{C}$. The concentration of DT-010 was set based on a previous study (20), which showed that $300 \mu \mathrm{M}$ DT-010 treatment alone may exert cytotoxicity. Therefore, a dose range of DT-010 between 10 and $100 \mu \mathrm{M}$ was chosen for the present study.

Cell viability was measured using MTT assay. To detect the effects of DT-010 on t-BHP-induced injury, DT-010 was first added and the cells were incubated for $1 \mathrm{~h}$ at $37^{\circ} \mathrm{C}$. Subsequently, cell supernatant was removed, and the cells were washed twice with Hanks' balanced salt solution (HBSS) to avoid drug interaction. After $6 \mathrm{~h}$ exposure to $300 \mu \mathrm{M}$ t-BHP at $37^{\circ} \mathrm{C}$, the cell supernatant was then removed before the cells were incubated with $100 \mu 10.5 \mathrm{mg} / \mathrm{ml} \mathrm{MTT}$ in the dark at $37^{\circ} \mathrm{C}$ for $4 \mathrm{~h}$. The crystals were dissolved by DMSO and the optical density at $570 \mathrm{~nm}$ was measured in each well. Cell viability was determined as previously described (20).

The activity of LDH in the cell supernatant was measured using the LDH kit according to the manufacturer's protocol.

For autophagy agonist and blocker treatment, cells were first pretreated with the autophagy agonist rapamycin (500 $\mathrm{nM})(23)$ or the autophagy blocker hydroxy-chloroquine $(50 \mu \mathrm{M})(24,25)$ for $1 \mathrm{~h}$ before being treated with DT-010 for $1 \mathrm{~h}$ and t-BHP for a further $6 \mathrm{~h}$. All treatments were performed at $37^{\circ} \mathrm{C}$. In between each treatment, cells were washed twice with HBSS to avoid drug interaction.

Cell apoptosis analysis. Hoechst 33342 nuclear staining was performed to assess cell apoptosis detection as described previously (20). Following DT-010 treatment for $1 \mathrm{~h}$, the cell supernatant was removed, and cells were washed twice with HBSS. Subsequently, the cells were treated with $300 \mu \mathrm{M}$ t-BHP for another $2 \mathrm{~h}$ at $37^{\circ} \mathrm{C}$. H9c2 cells were subsequently dyed with Hoechst 33342 at a final concentration of $5 \mu \mathrm{g} / \mathrm{ml}$ at $37^{\circ} \mathrm{C}$ in the dark for $20 \mathrm{~min}$ and then observed under an inverted fluorescence microscope (magnification, $\mathrm{x} 400$ ). After Hoechst staining, normal cells stain dark blue with homogeneous fluorescence intensity whereas apoptotic cells are characterized by shrunken and irregular nuclear shapes, in addition to broken and condensed chromatin. Cell apoptosis measurement was repeated three times independently, with 6-8 fields of view in each group randomly photographed. The apoptosis rate was calculated using ImageJ software version 1.44p (bundled with Java v1.6.0_20; National 
<smiles>O=C(O)[C@H](O)Cc1ccc(O)c(O)c1</smiles>

DSS<smiles>Cc1nc(C)c(C)nc1C</smiles>

TMP<smiles>C=CCC(CC=C)(OC(=O)[C@@H](Cc1ccc(O)c(O)c1)OC(C)=O)c1nc(C)c(C)nc1C</smiles>

DT-010

Figure 1. Structural formula of DSS, TMP and DT-010. DSS, danshensu; TMP, tetramethypyrazin.

Institutes of Health) using the following formula: Apoptotic rate $=$ (number of apoptotic cells/number of total cells) $\times 100 \%$.

Autophagic flow analysis. Autophagic flow was evaluated using the mRFP-GFP-LC3 assay. H9c2 cells were first seeded onto the laser confocal culture dishes at a density of $5 \times 10^{3}$ cells/dish. After reaching $30 \%$ confluence, cells were transfected with the mRFP-GFP-LC3 adenovirus $\left(1.58 \times 10^{10} \mathrm{pfu} / \mathrm{ml}\right)$ at $1,500 \mathrm{MOI}$ for $36 \mathrm{~h}$. After another $48 \mathrm{~h}$ of culture in complete DMEM, the cells were treated with DT-010 for $1 \mathrm{~h}$ and $300 \mu \mathrm{M} \mathrm{t}$-BHP for $2 \mathrm{~h}$ at $37^{\circ} \mathrm{C}$. Finally, cells were fixed with $4 \%$ paraformaldehyde at room temperature for $15 \mathrm{~min}$ and then observed under a laser confocal microscope (magnification, x630). Autophagic flux was visualized and analyzed via fluorescence imaging of autophagosomes and autolysosomes. Yellow $\left(\mathrm{GFP}^{+} / \mathrm{mRFP}^{+}\right)$ and red $\left(\mathrm{GFP}^{-} / \mathrm{mRFP}^{+}\right)$puncta indicate the presence of autophagosomes and autolysosomes, respectively.

$A O$ and MDC staining. $\mathrm{AO}$ and MDC staining are commonly used to measure autophagosome vesicle formation $(26,27)$. After $1 \mathrm{~h}$ treatment with $30 \mu \mathrm{M}$ DT-010 and another $2 \mathrm{~h}$ $300 \mu \mathrm{M}$ t-BHP treatment at $37^{\circ} \mathrm{C}$, cells were gently rinsed with HBSS and stained with $1 \mu \mathrm{M} \mathrm{AO}$ and $50 \mathrm{nM}$ MDC for $10 \mathrm{~min}$ in the incubator at $37^{\circ} \mathrm{C}$. Cells were observed under an inverted fluorescence microscope (magnification, x200), where six-eight fields of view were randomly imaged in each group.

Western blotting. Proteins were extracted from H9c2 cells using RIPA lysis buffer (Beyotime Institute of Biotechnology). Proteins were quantified with a BCA protein assay kit (Beyotime Institute of Biotechnology) and $30 \mu \mathrm{g}$ denatured protein from each sample was subjected to 10 or $12 \%$ SDS-PAGE. Proteins were then transferred onto PVDF membranes. Following blocking of the membrane with $5 \%$ skimmed milk at room temperature for $2 \mathrm{~h}$, immunoblotting was performed by incubation with specific primary antibodies $(1: 1,000)$ at $4^{\circ} \mathrm{C}$ overnight. After washing, horseradish peroxidase-labeled secondary antibodies (1:2,000) were added and the membranes were incubated at room temperature for $2 \mathrm{~h}$. An ECL detection kit (cat. no. P0018S; Beyotime Institute of Biotechnology) was utilized for protein detection. The optical density of the protein bands was analyzed using the Carestream MI SE system (4,000 MM PRO system; Carestream Health, Inc.).

Statistical analysis. Results are presented as the mean \pm SD of at least three experimental repeats. All data were analyzed using GraphPad Prism software (version 6.01; GraphPad Software, Inc.). Significant differences were determined using one-way ANOVA followed by Tukey's test. $\mathrm{P}<0.05$ was considered to indicate a statistically significant difference.

\section{Results}

DT-010 protects cardiomyocytes against oxidative stress damage. $\mathrm{H} 9 \mathrm{c} 2$ cardiomyocytes were first exposed to t-BHP to mimic ischemia-reperfusion-induced cellular oxidative stress damage. DT-010 alone did not induce cytotoxicity in H9c2 cells (Figs. 2A, B and S1). Compared with cells in the control group, t-BHP exposure significantly decreased cell viability whilst significantly increasing LDH release (Fig. 2A and B). DT-010 pretreatment dose-dependently restored cell viability and reduced LDH release compared with cells in the t-BHP-only group (Fig. 2A and B), suggesting a cardioprotective effect of DT-010 which was observed at 30 and $100 \mu \mathrm{M}$. Subsequently, Hoechst 33342 staining was performed for cell apoptosis detection. Normal cells were stained dark blue with homogeneous fluorescence intensity whereas apoptotic cells were characterized by shrunken and irregular nuclear shapes, coupled with condensed chromatin (Fig. 2C and D). The level of apoptosis in $\mathrm{H} 9 \mathrm{c} 2$ cells increased significantly following t-BHP exposure compared with that in the control group, but was significantly lower in cells treated with DT-010. This observation suggests a protective effect of DT-010 against apoptosis induced by t-BHP (Fig. 2C and D).

DT-010 inhibits oxidative stress-induced autophagy in cardiomyocytes. LC3 protein is a hallmark protein of autophagy induction (26). When autophagy is initiated, LC3-I, the cytosolic form of LC3 is converted into the autophagosomal membrane LC3-II form via enzymatic digestion (26). Therefore, increased LC3-II/LC3-I ratios can be used to indicate autophagosome formation (26). t-BHP treatment significantly increased the LC3-II/LC3-I ratio compared with cells in the control group, which was significantly prevented by DT-010 pretreatment (Fig. 3A), suggesting that DT-010 can inhibit oxidative stress-induced autophagy in cardiomyocytes. Furthermore, AO and MDC staining demonstrated that DT-010 may inhibit autophagy induced by oxidative stress (Fig. 3B). $\mathrm{AO}$ and MDC are two commonly used specific agents for the assessment of autophagosomes, acidic endosomes and lysosomes. Acidic vesicular organelles including autophagolysosomes can be stained by AO, while MDC represents widely used fluorescent probes that preferentially accumulate 

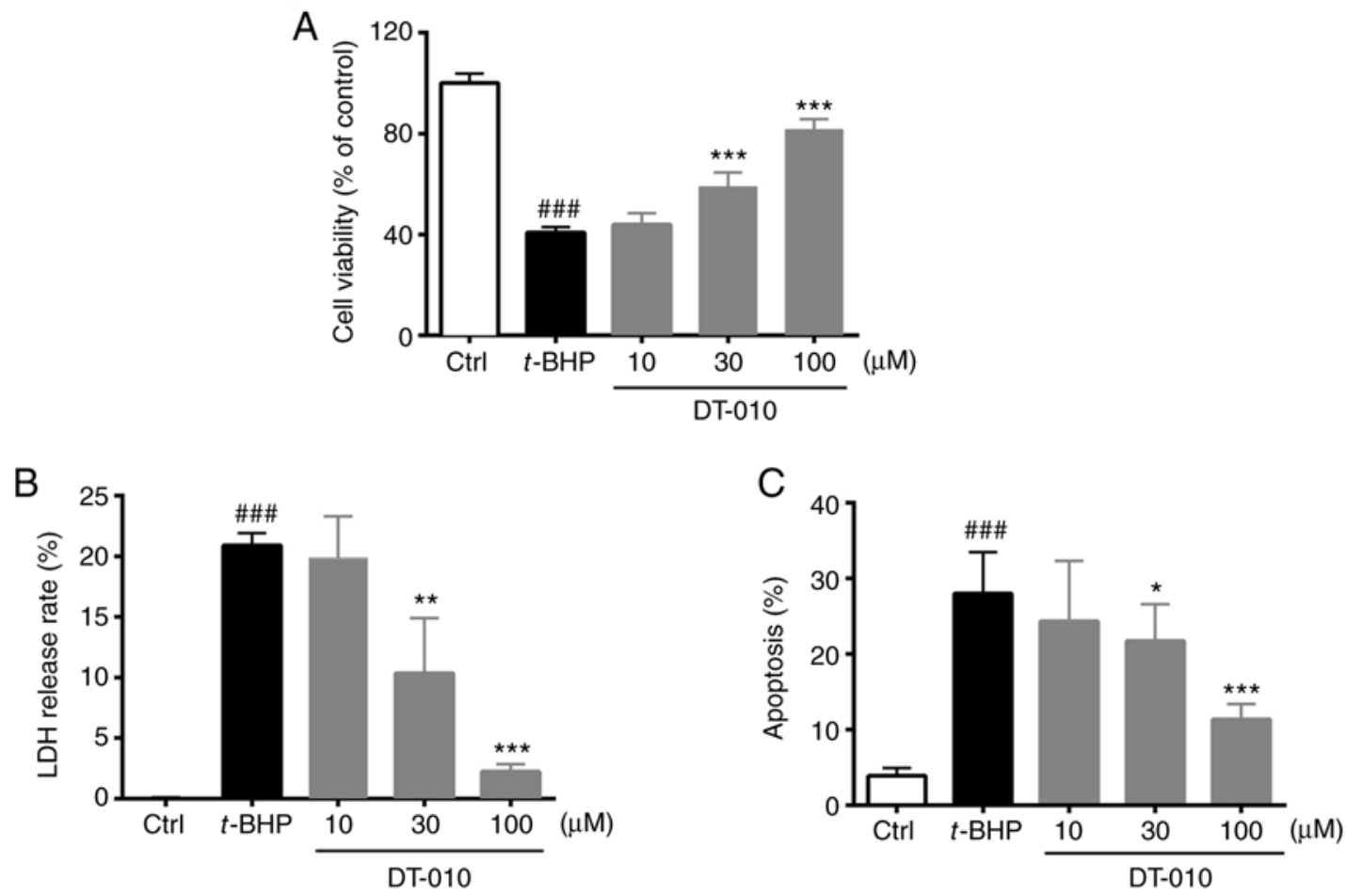

D

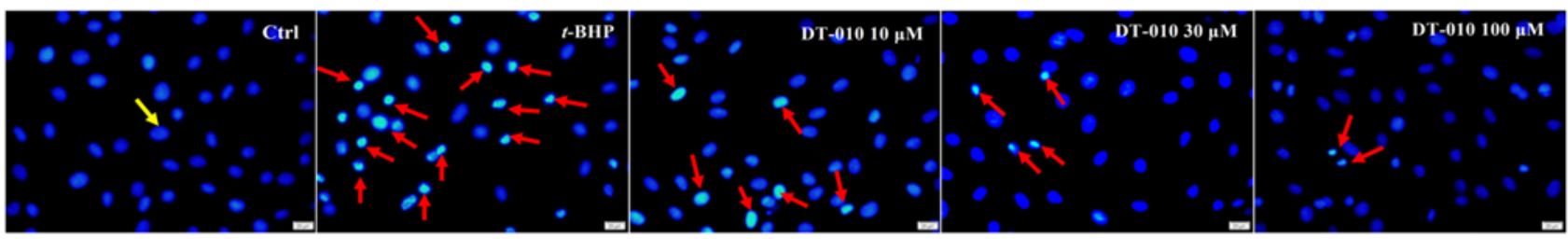

Figure 2. DT-010 protects cardiomyocytes against t-BHP-induced injury. (A) Cell viability and (B) lactate dehydrogenase release were measured. Data are presented as the mean \pm SD from six experimental repeats. (C) Percentage of apoptotic cells was measured, which was calculated from (D) representative Hoechst 33342 staining images. Yellow and red arrows indicate normal and apoptotic cells, respectively. Scale bars, $20 \mu \mathrm{m}$. Data are presented as the mean \pm SD from three experimental repeats. ${ }^{\# \# "} \mathrm{P}<0.001$ vs. Ctrl; ${ }^{*} \mathrm{P}<0.05,{ }^{* *} \mathrm{P}<0.01$ and ${ }^{* * *} \mathrm{P}<0.001$ vs. t-BHP. t-BHP, tert-butylhydroperoxide; Ctrl, control; $\mathrm{LDH}$, lactate dehydrogenase.

in autophagic vacuoles (26-28). Following t-BHP treatment, the number of orange-red $\mathrm{AO}$ stained particles and the extent of MDC green fluorescence were observed to be increased. By contrast, DT-010 pre-treatment markedly reduced the formation of autophagosome vesicles as indicated by $\mathrm{AO}$ and MDC staining.

Autophagic flux is characterized by sequential conversion of autophagosomes into autolysosomes (26). The number of autolysosomes (red puncta; $\mathrm{GFP}^{-} / \mathrm{mRFP}^{+}$) were found to be markedly increased in cells in the t-BHP-treated group compared with that in the control group, while autophagosomes (yellow puncta; $\mathrm{GFP}^{+} / \mathrm{mRFP}^{+}$) exhibited no difference between the two groups. However, in cells pre-treated with DT-010, the numbers of autolysosomes $\left(\mathrm{GFP}^{-} / \mathrm{mRFP}^{+}\right)$were decreased compared with that in the t-BHP-treated group (Fig. 3C). Therefore, these data suggest that DT-010 may inhibit autophagic flow induced by t-BHP in cardiomyocytes.

DT-010 prevents oxidative damage induced by $t$-BHP by inhibiting of autophagy. MTT assay results revealed that $\mathrm{t}$-BHP treatment significantly decreased cell viability (Fig. 4). Treatment with the autophagy agonist rapamycin treatment significantly aggravated cell injury induced by
$t$-BHP further (Fig. 4A), whilst autophagy blocker chloroquine treatment significantly attenuated t-BHP-induced cell injury (Fig. 4B). These data indicate that autophagy may be involved in t-BHP-induced cardiomyocyte damage. DT-010 pretreatment significantly prevented t-BHP-induced cell damage (Fig. 4), which was partially but significantly abolished by rapamycin (Fig. 4A) and significantly improved by hydroxy-chloroquine treatment (Fig. 4B). These results suggest that the cardioprotective effects of DT-010 could be mediated by inhibiting autophagy.

DT-010 attenuates autophagy via the AMPK-mTOR-UlkI signaling pathway.t-BHP treatment increased the LC3-II/LC3-I ratio whilst downregulating the expression levels of p62 compared with those in cells in the control group (Fig. 5). However, DT-010 pretreatment significantly reduced the LC3-II/LC3-I ratio and significantly increased the expression levels of p62 compared with those in the t-BHP group (Fig. 5A). AMPK-mTOR-Ulk1/2 has been previously reported to be one of the signaling pathways that regulate autophagy (29-32). Therefore, the present study further measured the expression levels of key components of the AMPK/mTOR/Ulk1 signaling pathway. t-BHP treatment significantly increased 
A

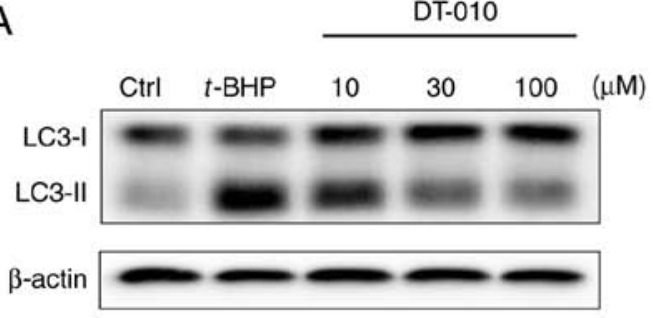

B

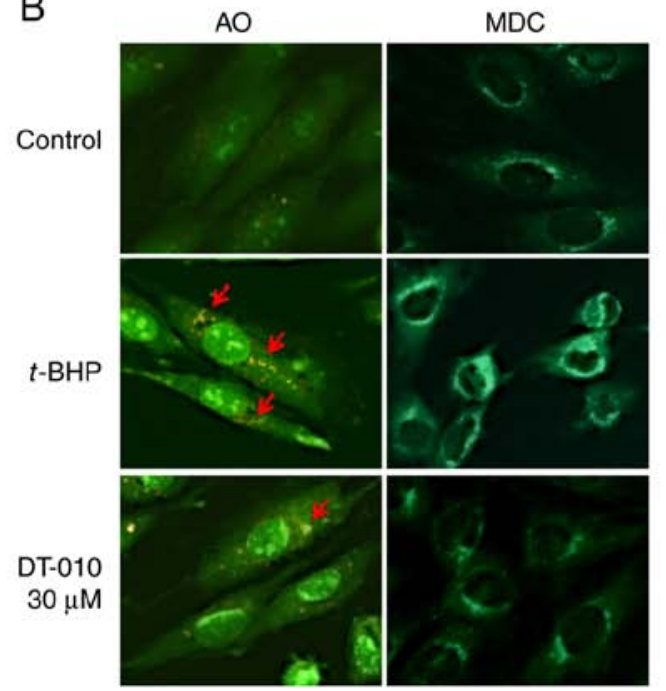

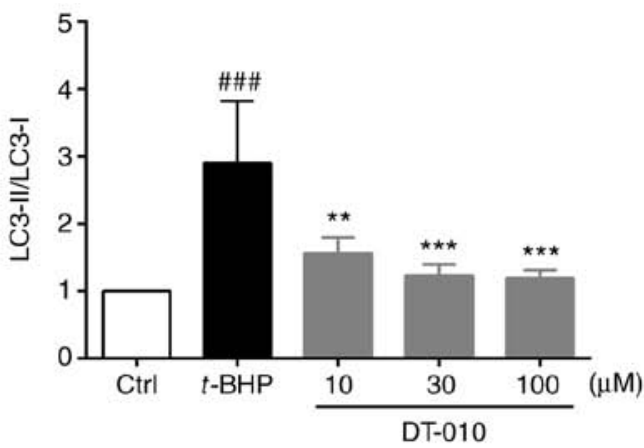

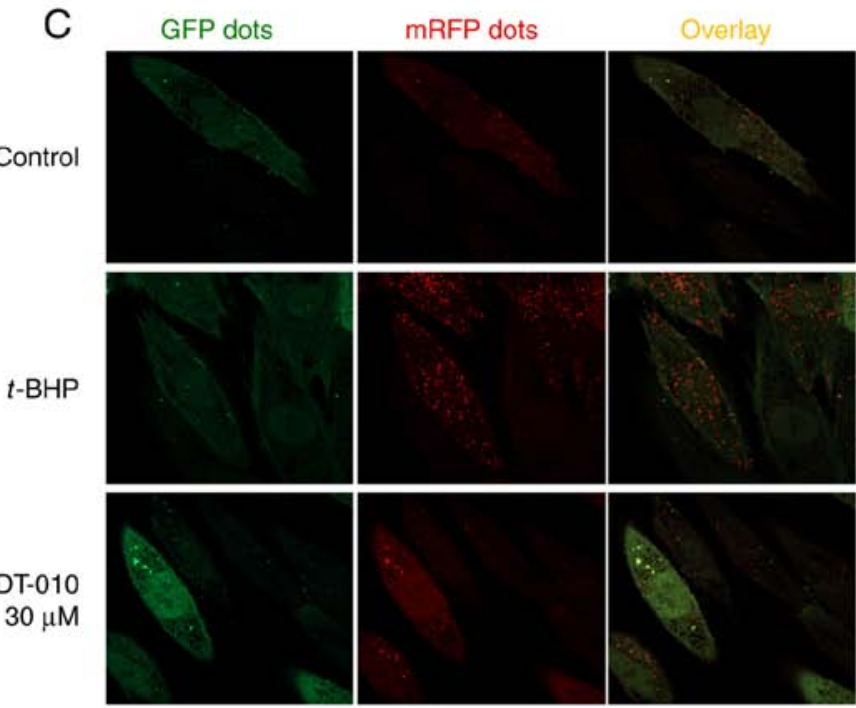

Figure 3. DT-010 inhibits oxidative stress-induced autophagy in cardiomyocytes. (A) Conversion of LC3-I into LC3-II in H9c2 cells was detected by western blotting and quantified. (B) H9c2 cells were stained using AO and MDC. Magnification, x200. Red arrows indicate acidic vesicular organelles including autophagolysosomes stained by AO. (C) H9c2 cells were transfected with the mRFP-GFP-LC3 adenovirus and LC3 puncta were observed. Magnification, x630. Data are presented as the mean \pm SD from four experimental repeats. ${ }^{\# \# \#} \mathrm{P}<0.001$ vs. Ctrl; ${ }^{* *} \mathrm{P}<0.01$ and ${ }^{* * * *} \mathrm{P}<0.001$ vs. t-BHP. t-BHP, tert-butylhydroperoxide; $\mathrm{AO}$, acridine orange; MDC, monodansylcadaverine; GFP, green fluorescent protein; mRFP, monomeric red fluorescent protein; LC3, microtubule-associated protein 1A/1B-light chain 3 .

A

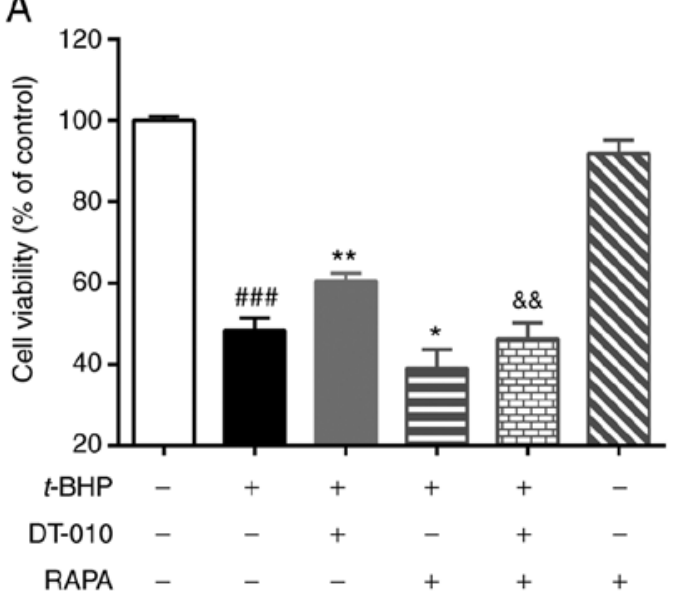

B

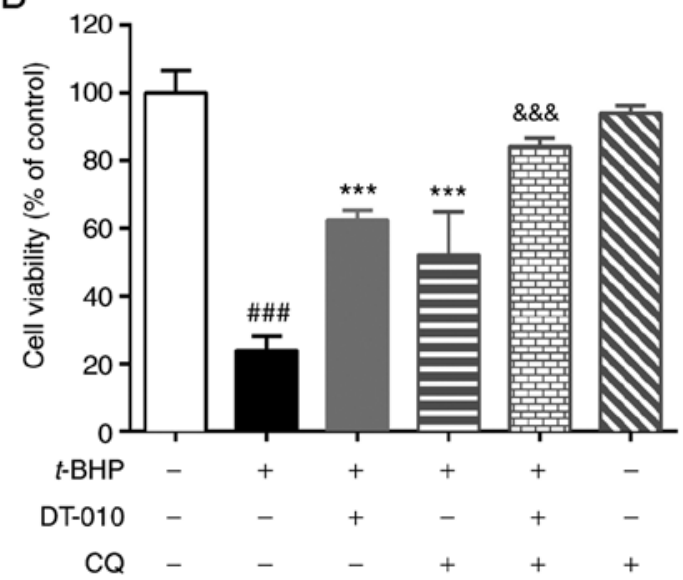

Figure 4. DT-010 protects cardiomyocytes from oxidative damage by inhibiting autophagy. (A) Autophagy agonist rapamycin and (B) autophagy blocker CQ were used to investigate the role of autophagy in the cardioprotective effects of DT-010. MTT assay was used for cell viability determination. Data are presented as the mean $\pm \mathrm{SD}$ from four experimental repeats. ${ }^{\# \# \#} \mathrm{P}<0.001$ vs. Untreated; ${ }^{*} \mathrm{P}<0.05,{ }^{* *} \mathrm{P}<0.01$ and ${ }^{* * *} \mathrm{P}<0.001$ vs. $\mathrm{t}-\mathrm{BHP} ;{ }^{\&} \mathrm{P}<0.01$ and \&\&\&P<0.001 vs. t-BHP + DT-010. CQ, hydroxy-chloroquine; RAPA, rapamycin; t-BHP, tert-butylhydroperoxide.

the levels of AMPK phosphorylation whilst downregulating the expression of total AMPK. By contrast, AMPK phosphorylation was significantly decreased whereas the total expression of AMPK was significantly increased in cells pre-treated with DT-010 compared with those in cells treated with t-BHP alone (Fig. 5B). The expression of total mTOR 
A
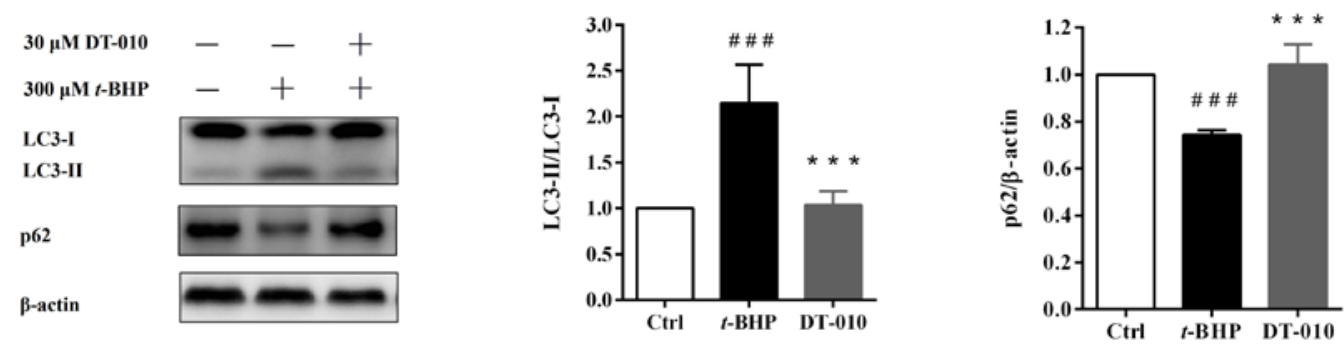

B
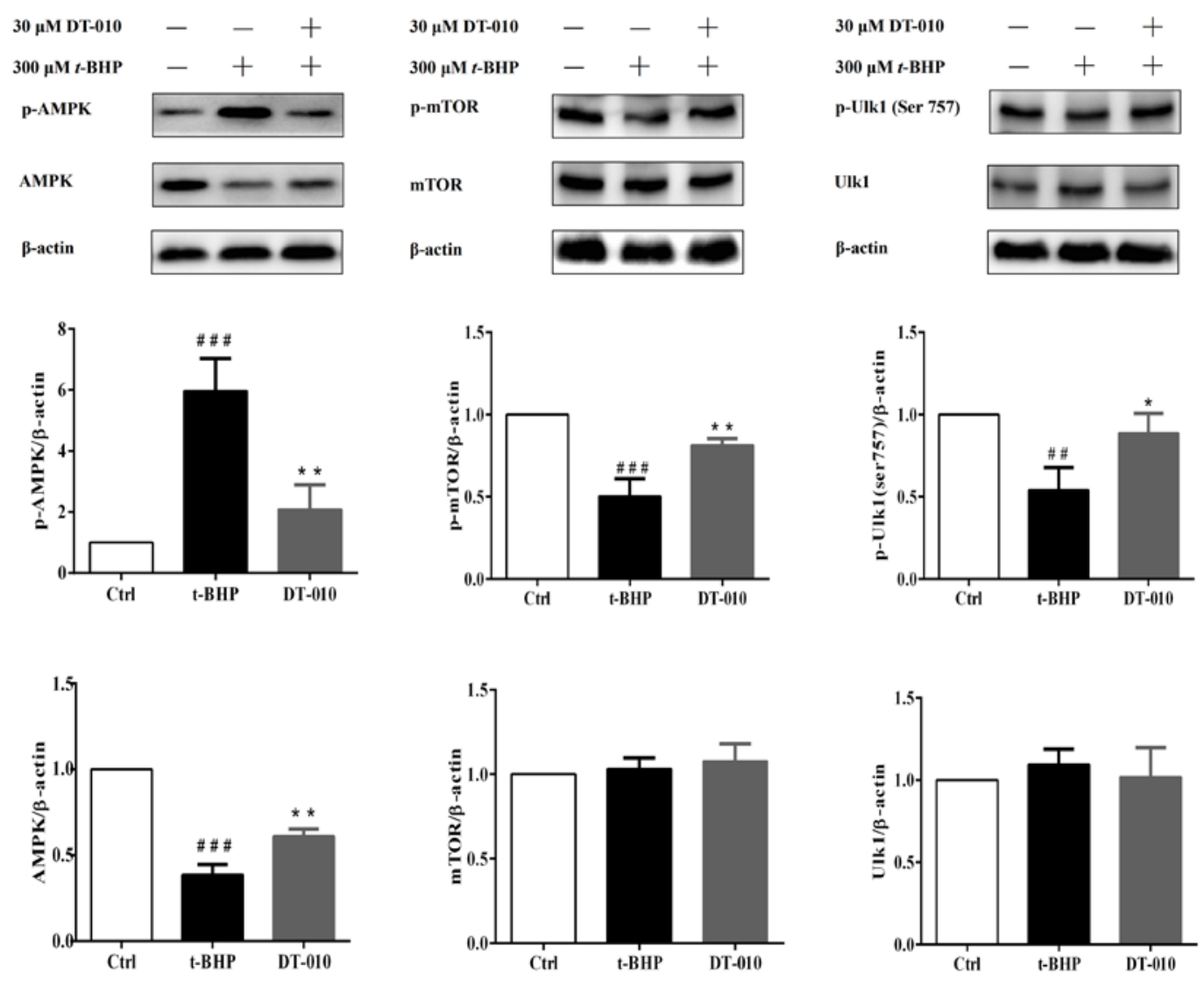

Figure 5. DT-010 inhibits autophagy via the AMPK/mTOR/Ulk1 signaling pathway. (A) LC3-I to LC3-II conversion and p62 expression were measured and quantified by western blotting. (B) Expression of total AMPK, mTOR and Ulk1 protein and their corresponding phosphorylation were measured by western blotting. Data are presented as the mean $\pm \mathrm{SD}$ from three experimental repeats. ${ }^{\# \#} \mathrm{P}<0.01$ and ${ }^{\# \# \#} \mathrm{P}<0.001$ vs. Ctrl; ${ }^{*} \mathrm{P}<0.05,{ }^{* *} \mathrm{P}<0.01$ and ${ }^{* * *} \mathrm{P}<0.001$ vs. t-BHP. AMPK, 5'-AMP-activated protein kinase; Ulk1, unc-51 like autophagy activating kinase 1; Ctrl, control; LC3, microtubule-associated protein 1A/1B-light chain 3; t-BHP, tert-butylhydroperoxide; p-, phosphorylated.

and Ulk1 proteins were not observed to be altered after t-BHP treatment or DT-010 pretreatment (Fig. 5B). However, t-BHP treatment significantly decreased the levels of mTOR and Ulk1 phosphorylation compared with cells in the control group (Fig. 5B), both of which were significantly prevented by DT-010 pretreatment (Fig. 5B). These results suggested that DT-010 may inhibit t-BHP-induced intracellular autophagy by inhibiting the AMPK-mTOR-Ulk1 signaling pathway.

\section{Discussion}

Based on the previously reported properties of DSS and TMP on the cardiovascular system, a large number of DSS and TMP conjugates was synthesized previously, following which their cardiovascular effects were screened in different models in vivo and in vitro (21). Previous structure-effect studies demonstrated that DT-010 is a compound that constitute DSS and TMP linked via an ester bond (22) and displayed better cardioprotective effects compared with DSS, TMP or a combination of the two (20-22,33-35). However, the mechanism underlying the cardioprotective effects of DT-010 remain unclear. Therefore, the present study explored the involvement of autophagy in the cardioprotective effect of DT-010 against oxidative stress injury. DT-010 may facilitate cardiomyocyte survival following t-BHP insult, implicating the cardioprotective effects of DT-010 reported in a previous study (20). DT-010 may be considered as a candidate for myocardial ischemia and reperfusion injury treatment.

Autophagy has been considered to be an important target for treating ischemia-reperfusion injury (5). A large number 
of studies have previously revealed that myocardial ischemia and reperfusion causes mass generation of ROS in cells, which triggers autophagy $(5,36,37)$. t-BHP is commonly used as an oxidative stress inducer due to its ability of producing free radicals (15). The present study demonstrated that t-BHP exposure stimulated autophagy formation in a manner that was associated with marked increases in the LC3-II/LC3-I ratio and autophagic flux. Furthermore, the expression of p62, an autophagy-specific metabolic substrate, was decreased following t-BHP treatment. DT-010 pretreatment was found to inhibit t-BHP-induced autophagy. Subsequently, rapamycin treatment prior to $\mathrm{t}-\mathrm{BHP}$ exposure was observed to aggravate t-BHP-mediated cell damage and autophagy. By contrast, hydroxy-chloroquine treatment attenuated cell damage and autophagy induced by t-BHP treatment, suggesting that t-BHP induced $\mathrm{H} 9 \mathrm{c} 2$ cell damage by activating autophagy. DT-010 attenuated t-BHP-induced cardiomyocyte injury by at least in part inhibiting autophagy, which was partially but significantly abolished by rapamycin and significantly improved by hydroxy-chloroquine treatment. At present, different physiological consequences of autophagy have been observed in myocardial ischemia reperfusion injury. Short-term and moderate activation of autophagy may confer beneficial effects by degrading dysfunctional or damaged proteins and organelles to promote cell survival, whilst a persistent elevation of autophagy may promote cell death (38-42). The duration and degree of ischemia and reperfusion are essential for the modulation of autophagy as a pharmacological strategy $(43,44)$. Therefore, a number of studies have reported that activation of autophagy could mitigate cardiac ischemia/reperfusion injury (45-47). However, other studies have also reported that inhibition of autophagy could alleviate cardiac ischemia/reperfusion injury $(42,48,49)$, which were consistent with results from the present study. In the future, the optimal time course of DT-010 treatment following myocardial ischemia and reperfusion injury in animal models should be investigated, such that more data is required prior to its proposed clinical use.

The regulation of signal transduction molecules and pathways involved in autophagy is highly complex. The AMPK-mTOR-Ulk1/2 pathway is an important signaling pathway that regulates autophagy (29-32,50-54). AMPK is a pivotal energy sensor for maintaining metabolic homeostasis, whereby AMPK is activated in cells suffering from ischemia and reperfusion during starvation (29). t-BHP-induced ROS accumulation causes oxidative stress and dysfunction in mitochondria and results in an energy crisis $(16,55,56)$, which can lead to the activation of AMPK. AMPK activation may result in the inactivation of $\mathrm{mTOR}$ and activation of Ulk1 via the phosphorylation of the Ser317 and Ser777 residues, thus promoting autophagy (57). Phosphorylation of Ulk1 Ser757 prevents Ulk1 activation and inhibits autophagy (57). It was previously reported that AMPK inhibitor Compound $\mathrm{C}$ could suppress AMPK/mTOR-mediated autophagy in a rat myocardial infarction model (58). The present study revealed that the levels of p-AMPK were increased whereas total AMPK protein expression was inhibited after t-BHP treatment. Following DT-010 pre-treatment, phosphorylation of AMPK was decreased, whilst total expression of AMPK was enhanced compared with cells treated with t-BHP alone. A previous study reported that the expression of total AMPK protein can be altered by various factors, such as menadione-induced oxidative stress (59). In the present study, it was therefore speculated that the expression of total AMPK protein may be inhibited by severe oxidative stress. However, the underlying mechanism of this phenomenon need to be investigated further. In addition, it was also found that the levels of p-mTOR and p-Ulk1 (Ser757) were decreased in cells in the t-BHP group, which could be prevented by DT-010 pre-treatment. Rapamycin treatment attenuated the cardioprotective effects of DT-010 against t-BHP-induced toxicity in $\mathrm{H} 9 \mathrm{c} 2$ cells. This observation suggested that DT-010 stimulated mTOR activity, leading to the suppression of autophagy initiation. A previous study revealed that DT-010 markedly elevated Akt phosphorylation, followed by the reduction in AMPK phosphorylation (inhibition) and subsequent phosphorylation and activation of mTOR within 10 min after treatment (21). Therefore, it can be concluded that DT-010 may inhibit t-BHP-induced autophagy in cardiomyocytes by inhibiting the AMPK-mTOR-Ulk1 signaling pathway, thereby preventing t-BHP-induced cardiomyocyte injury. Previous studies also revealed the potent antioxidative effects of DT-010 (20,22). DT-010 was found to eliminate a number of free radical species $\left(\bullet \mathrm{O}_{2}^{-}, \bullet \mathrm{OH}\right.$ and $\left.\mathrm{ONOO}^{-}\right)$ induced by t-BHP and increases the expression levels of cellular redox-related proteins, including PGC- $1 \alpha, \mathrm{Nrf} 2$, and HO-1 (20). Since oxidative stress caused by ROS is a potent inducer of autophagy (16), scavenging of ROS and alleviation of oxidative stress by DT-010 could also contribute to its inhibitory effects on t-BHP-induced autophagy.

In summary, DT-010 was found to protect cardiomyocytes against oxidative stress injury by inhibiting autophagy through the AMPK-mTOR-Ulk1 signaling pathway. The findings of the present study provided evidence for the use of autophagy regulators in therapeutic strategies for myocardial ischemia and reperfusion injury. DT-010 may be a promising candidate for myocardial ischemia-reperfusion injury therapy.

\section{Acknowledgements}

Not applicable.

\section{Funding}

The present study was supported by grants from the National Natural Science Foundation of China (grant no. 81703509), and Science and Technology Planning Project of Guangdong Province, China (grant no. 2015A020211015).

\section{Availability of data and materials}

The datasets used and/or analyzed during the present study are available from the corresponding author on reasonable request. Compound DT-010 is available with the agreement of the corresponding author.

\section{Authors' contributions}

LW and LS conceived, designed and supervised the whole study. JL, CX and $\mathrm{HH}$ performed the experiments, and further analyzed and interpreted the data. YW, PY, YS, and LX 
provided some professional help during the experiment. CX, LW, YW and LS wrote and revised the manuscript. All authors read and approved the final manuscript.

\section{Ethics approval and consent to participate}

Not applicable.

\section{Patient consent for publication}

Not applicable.

\section{Competing interests}

Yuqiang Wang, Pei Yu, Luchen Shan and Yewei Sun are inventors of the patent covering DT-010 (patent no. CN105294666A) and have financial interest in Changzhou Magpie Pharmaceuticals, Inc. (Changzhou, China), which is developing DT-010 as a therapeutic agent.

\section{References}

1. Mendis S, Puska P and Norrving B, World Health Organization, World Heart Federation: Global atlas on cardiovascular disease prevention and control. Mendis S and Puska P (eds). WHO, Geneva, pp1-155, 2011.

2. Kalogeris T, Baines CP, Krenz $M$ and Korthuis RJ: Ischemia/reperfusion. Compr Physiol 7: 113-170, 2016.

3. Li T, Su Y, Yu X, Mouniir DSA, Masau JF, Wei X and Yang J: Trop2 guarantees cardioprotective effects of cortical bone-derived stem cells on myocardial ischemia/reperfusion injury. Cell Transplant 27: 1256-1268, 2018.

4. Matsui Y, Takagi H, Qu X, Abdellatif M, Sakoda H, Asano T, Levine B and Sadoshima J: Distinct roles of autophagy in the heart during ischemia and reperfusion: Roles of AMP-activated protein kinase and Beclin 1 in mediating autophagy. Circ Res 100: 914-922, 2007.

5. Hamacher-Brady A, Brady NR, Logue S, Sayen MR, Jinno M, Kirshenbaum L, Gottlieb RA and Gustafsson AB: Response to myocardial ischemia/reperfusion injury involves Bnip3 and autophagy. Cell Death Differ 14: 146-157, 2007.

6. Valentim L, Laurence KM, Townsend PA, Carroll CJ, Soond S, Scarabelli TM, Knight RA, Latchman DS and Stephanou A: Urocortin inhibits Beclin1-mediated autophagic cell death in cardiac myocytes exposed to ischaemia/reperfusion injury. J Mol Cell Cardiol 40: 846-852, 2006.

7. Yan L, Sadoshima J, Vatner DE and Vatner SF: Autophagy: A novel protective mechanism in chronic ischemia. Cell Cycle 5: 1175-1177, 2006

8. Yan L, Vatner DE, Kim SJ, Ge H, Masurekar M, Massover WH, Yang G, Matsui Y, Sadoshima J and Vatner SF: Autophagy in chronically ischemic myocardium. Proc Natl Acad Sci USA 102: 13807-13812, 2005.

9. Li X, Huang Q, Wang M, Yan X, Song X, Ma R, Jiang R, Zhao D and Sun L: Compound $\mathrm{K}$ inhibits autophagy-mediated apoptosis through activation of the PI3K-Akt signaling pathway thus protecting against ischemia/reperfusion injury. Cell Physiol Biochem 47: 2589-2601, 2018.

10. Przyklenk K, Dong Y, Undyala VV and Whittaker P: Autophagy as a therapeutic target for ischaemia/reperfusion injury? Concepts, controversies, and challenges. Cardiovasc Res 94: 197-205, 2012.

11. Araujo TF, Cordeiro AV, Vasconcelos DAA, Vitzel KF and Silva VRR: The role of cathepsin B in autophagy during obesity: A systematic review. Life Sci 209: 274-281, 2018.

12. Ravikumar B, Sarkar S, Davies JE, Futter M, Garcia-Arencibia M, Green-Thompson ZW, Jimenez-Sanchez M, Korolchuk VI, Lichtenberg M, Luo S, et al: Regulation of mammalian autophagy in physiology and pathophysiology. Physiol Rev 90: 1383-1435, 2010.

13. Zhao W, Feng H, Sun W, Liu K, Lu JJ and Chen X: Tert-butyl hydroperoxide (t-BHP) induced apoptosis and necroptosis in endothelial cells: Roles of NOX4 and mitochondrion. Redox Biol 11: 524-534, 2017.
14. Zhao Y, Zhang F, Zhao X, Yuan W, Zhang J and Wang Y: Shenmai injection protects mitochondria from oxidative injury in myocardial cells and its mechanism. Zhejiang Da Xue Xue Bao Yi Xue Ban 47: 507-513, 2018 (In Chinese).

15. Silva JP, Sardao VA, Coutinho OP and Olveira PJ: Nitrogen compounds prevent h9c2 myoblast oxidative stress-induced mitochondrial dysfunction and cell death. Cardiovasc Toxicol 10: 51-65, 2010.

16. Lu D, Zhu LH, Shu XM, Zhang CJ, Zhao JY, Qi RB, Wang HD and Lu DX: Ginsenoside Rg1 relieves tert-Butyl hydroperoxide-induced cell impairment in mouse microglial BV2 cells. J Asian Nat Prod Res 17: 930-945, 2015.

17. Li Z, Jiang T, Lu Q, Xu K, He J, Xie L, Chen Z, Zheng Z, Ye L, $\mathrm{Xu} \mathrm{k}$, et al: Berberine attenuated the cytotoxicity induced by t-BHP via inhibiting oxidative stress and mitochondria dysfunction in PC-12 cells. Cell Mol Neurobiol 40: 587-602, 2020.

18. Fan G, Yu J, Asare PF, Wang L, Zhang H, Zhang B, Zhu Y and Gao X: Danshensu alleviates cardiac ischaemia/reperfusion injury by inhibiting autophagy and apoptosis via activation of mTOR signalling. J Cell Mol Med 20: 1908-1919, 2016.

19. Zhigang P, Huishan P, Guangchun J and Xiangshan L: An experimental study of ligustrazine on ischemic myocardial protection and scavenging oxygen free radicals. Chin Wild Plant Resou 5: $21,2000$.

20. Zhang X, Hu H, Luo J, Deng H, Yu P, Zhang Z, Zhang G, Shan L and Wang Y: A novel danshensu-tetramethylpyrazine conjugate DT-010 provides cardioprotection through the PGC- $1 \alpha / \mathrm{Nrf} 2 / \mathrm{HO}-1$ pathway. Biol Pharm Bull 40: 1490-1498, 2017.

21. Tang F, Zhou X, Wang L, Shan L, Li C, Zhou H, Lee SM and Hoi MP: A novel compound DT-010 protects against doxorubicin-induced cardiotoxicity in zebrafish and H9c2 cells by inhibiting reactive oxygen species-mediated apoptotic and autophagic pathways. Eur J Pharmacol 820: 86-96, 2018.

22. Wang Y, Zhang X, Xu C, Zhang G, Zhang Z, Yu P, Shan L, Sun Y and Wang Y: Synthesis and biological evaluation of danshensu and tetramethylpyrazine conjugates as cardioprotective agents. Chem Pharm Bull (Tokyo) 65: 381-388, 2017.

23. Shi X, Zhu H, Zhang Y, Zhou M, Tang D and Zhang H: XuefuZhuyu decoction protected cardiomyocytes against hypoxia/reoxygenation injury by inhibiting autophagy. BMC Complement Altern Med 17: 325, 2017.

24. Zuo Y, Zhang J, Cheng X, Li J, Yang Z, Liu X, Gu E and Zhang Y: Enhanced autophagic flux contributes to cardioprotection of remifentanil postconditioning after hypoxia/reoxygenation injury in H9c2 cardiomyocytes. Biochem Biophys Res Commun 514: 953-959, 2019.

25. Zhao M, Sun L, Yu XJ, Miao Y, Liu JJ, Wang H, Ren J and Zang WJ: Acetylcholine mediates AMPK-dependent autophagic cytoprotection in H9c2 cells during hypoxia/reoxygenation injury. Cell Physiol Biochem 32: 601-613, 2013.

26. Mizushima N: Methods for monitoring autophagy. Int J Biochem Cell Biol 36: 2491-2502, 2004.

27. Chang CH, Lee CY, Lu CC, Tsai FJ, Hsu YM, Tsao JW, Juan YN, Chiu HY, Yang JS and Wang CC: Resveratrol-induced autophagy and apoptosis in cisplatin-resistant human oral cancer CAR cells: A key role of AMPK and Akt/mTOR signaling. Int J Oncol 50: 873-882, 2017.

28. Liu F, Gao S, Yang Y, Zhao X, Fan Y, Ma W, Yang D, Yang A and $\mathrm{Yu}$ Y: Curcumin induced autophagy anticancer effects on human lung adenocarcinoma cell line A549. Oncol Lett 14: 2775-2782, 2017.

29. Alers S, Löffler AS, Wesselborg S and Stork B: Role of AMPK-mTOR-Ulk1/2 in the regulation of autophagy: Cross talk, shortcuts, and feedbacks. Mol Cell Biol 32: 2-11, 2012.

30. Ansari MY, Ahmad N and Haqqi TM: Butein activates autophagy through AMPK/TSC2/ULK1/mTOR pathway to inhibit IL-6 expression in IL-1 $\beta$ stimulated human chondrocytes. Cell Physiol Biochem 49: 932-946, 2018.

31. Zhang Y and Miao JM: Ginkgolide K promotes astrocyte proliferation and migration after oxygen-glucose deprivation via inducing protective autophagy through the AMPK/mTOR/ULK1 signaling pathway. Eur J Pharmacol 832: 96-103, 2018.

32. Cai ZY, Yang B, Shi YX, Zhang WL, Liu F, Zhao W and Yang MW: High glucose downregulates the effects of autophagy on osteoclastogenesis via the AMPK/mTOR/ULK1 pathway. Biochem Biophys Res Commun 503: 428-435, 2018.

33. Cui G, Shan L, Hung M, Lei S, Choi I, Zhang Z, Yu P, Hoi P, Wang Y and Lee SM: A novel Danshensu derivative confers cardioprotection via PI3K/Akt and Nrf2 pathways. Int J Cardiol 168: 1349-1359, 2013. 
34. Cui Q, Chen Y, Zhang M, Shan L, Sun Y, Yu P, Zhang G, Wang D, Zhao Z, Xu Q, et al: Design, synthesis, and preliminary cardioprotective effect evaluation of danshensu derivatives. Chem Biol Drug Des 84: 282-291, 2014.

35. Xu CJ, Deng HX, Chen HY, Cui QB, Shan LC, Yu P, Sun YW and Wang YQ: Design, synthesis and biological evaluations of novel conjugates of Danshensu, tetramethylpyrazine and hydrogen sulfide donors as cardioprotective agents. Asian J Chem 28: $2555-2561,2016$

36. Lemasters JJ, Nieminen AL, Qian T, Trost LC, Elmore SP Nishimura Y, Crowe RA, Cascio WE, Bradham CA,Brenner DA and Herman B: The mitochondrial permeability transition in cell death: A common mechanism in necrosis, apoptosis and autophagy. Biochim Biophys Acta 1366: 177-196, 1998.

37. Huang KY, Wang JN, Zhou YY, Wu SZ, Tao LY, Peng YP Que JQ, Xue YJ and Ji KT: Antithrombin III alleviates myocardia ischemia/reperfusion injury by inhibiting excessive autophagy in a phosphoinositide 3-kinase/Akt-dependent manner. Front Pharmacol 10: 516, 2019.

38. Zhang YW, Shi J, Li YJ and Wei L: Cardiomyocyte death in doxorubicin-induced cardiotoxicity. Arch Immunol Ther Exp (Warsz) 57: 435-445, 2009.

39. Wang D, Yu W, Liu Y, Zhong G, Zhao Z, Yan X and Liu Q: Roles of autophagy in ischemic heart diseases and the modulatory effects of Chinese herbal medicine. Am J Chin Med 45: 1401-1419, 2017.

40. Hao M, Zhu S, Hu L, Zhu H, Wu X and Li Q: Myocardial ischemic postconditioning promotes autophagy against ischemia reperfusion injury via the activation of the nNOS/AMPK/mTOR pathway. Int J Mol Sci 18: 614, 2017.

41. Gao C, Wang R, Li B, Guo Y, Yin T, Xia Y, Zhang F, Lian K, Liu Y, Wang H, et al: TXNIP/Redd1 signalling and excessive autophagy: A novel mechanism of myocardial ischaemia/reperfusion injury in mice. Cardiovasc Res 116: 645-657, 2020.

42. Li X, Hu X, Wang J, Xu W, Yi C, Ma R and Jiang H: Inhibition of autophagy via activation of PI3K/Akt/mTOR pathway contributes to the protection of hesperidin against myocardial ischemia/reperfusion injury. Int J Mol Med 42: 1917-1924, 2018.

43. Yang SS, Liu YB, Yu JB, Fan Y, Tang SY, Duan WT, Wang Z, Gan RT and Yu B: Rapamycin protects heart from ischemia/reperfusion injury independent of autophagy by activating PI3 kinase-Akt pathway and mitochondria K(ATP) channel. Pharmazie 65: 760-765, 2010.

44. Xu Q, Li X, Lu Y, Shen L, Zhang J, Cao S, Huang X, Bin J and Liao Y: Pharmacological modulation of autophagy to protect cardiomyocytes according to the time windows of ischaemia/reperfusion. Br J Pharmacol 172: 3072-3085, 2015.

45. Qu X, Chen X, Shi Q, Wang X, Wang D and Yang L: Resveratrol alleviates ischemia/reperfusion injury of diabetic myocardium via inducing autophagy. Exp Ther Med 18: 2719-2725, 2019.

46. Yang J, He J, Ismail M, Tweeten S, Zeng F, Gao L, Ballinger S, Young M, Prabhu SD, Rowe GC, et al: HDAC inhibition induces autophagy and mitochondrial biogenesis to maintain mitochondrial homeostasis during cardiac ischemia/reperfusion injury. J Mol Cell Cardiol 130: 36-48, 2019.

47. Wang F, Pulinilkunnil T, Flibotte S, Nislow C, Vlodavsky I, Hussein B and Rodrigues B: Heparanase protects the heart against chemical or ischemia/reperfusion injury. J Mol Cell Cardiol 131: 29-40, 2019.
48. Wang G, Dai G, Song J, Zhu M, Liu Y, Hou X, Ke Z, Zhou Y, Qiu H, Wang F, et al: Lactone component from ligusticum chuanxiong alleviates myocardial ischemia injury through inhibiting autophagy. Front Pharmacol 9: 301, 2018.

49. Wu S, Chang G, Gao L, Jiang D, Wang L, Li G, Luo X, Qin S, Guo $\mathrm{X}$ and Zhang D: Trimetazidine protects against myocardial ischemia/reperfusion injury by inhibiting excessive autophagy. J Mol Med (Berl) 96: 791-806, 2018.

50. Xi X, Zou C, Ye Z, Huang Y, Chen T and Hu H: Pioglitazone protects tubular cells against hypoxia/reoxygenation injury through enhancing autophagy via AMPK-mTOR signaling pathway. Eur J Pharmacol 863: 172695, 2019.

51. Yang Y, Li N, Chen T, Zhang C, Liu L, Qi Y and Bu P: Trimetazidine ameliorates sunitinib-induced cardiotoxicity in mice via the AMPK/mTOR/autophagy pathway. Pharm Biol 57: 625-631, 2019.

52. Dong Y, Chen H, Gao J, Liu Y, Li J and Wang J: Molecular machinery and interplay of apoptosis and autophagy in coronary heart disease. J Mol Cell Cardiol 136: 27-41, 2019.

53. Yan J, Yan JY, Wang YX, Ling YN, Song XD, Wang SY, Liu HQ, Liu QC, Zhang Y, Yang PZ, et al: Spermidine-enhanced autophagic flux improves cardiac dysfunction following myocardial infarction by targeting the AMPK/mTOR signalling pathway. Br J Pharmacol 176: 3126-3142, 2019.

54. Wang L, Yuan D, Zheng J, Wu X, Wang J, Liu X, He Y, Zhang C, Liu C, Wang T and Zhou Z: Chikusetsu saponin IVa attenuates isoprenaline-induced myocardial fibrosis in mice through activation autophagy mediated by AMPK/mTOR/ULK1 signaling. Phytomedicine 58: 152764, 2019.

55. Rabinovitc RC, Samborska B, Faubert B, Ma EH, Gravel SP, Andrzejewski S, Raissi TC, Pause A, St-Pierre J and Jones RG: AMPK maintains cellular metabolic homeostasis through regulation of mitochondrial reactive oxygen species. Cell Rep 21: 1-9, 2017.

56. Zhang T, Liu J, Tong Q and Lin L: SIRT3 acts as a positive autophagy regulator to promote lipid mobilization in adipocytes via activating AMPK. Int J Mol Sci 21: 372, 2020.

57. Kim J, Kundu M, Viollet B and Guan KL: AMPK and mTOR regulate autophagy through direct phosphorylation of Ulk1. Nat Cell Biol 13: 132-141, 2011.

58. Ren PH, Zhang ZM, Wang P, Zhu HP and Li ZQ: Yangxinkang tablet protects against cardiac dysfunction and remodelling after myocardial infarction in rats through inhibition of AMPK/mTOR-mediated autophagy. Pharm Biol 58: 321-327, 2020.

59. Jin Q, Jhun BS, Lee SH, Lee J, Pi Y, Cho YH, Baik HH and Kang I: Differential regulation of phosphatidylinositol 3-kinase/Akt, mitogen-activated protein kinase, and AMP-activated protein kinase pathways during menadione-induced oxidative stress in the kidney of young and old rats. Biochem Biophys Res Commun 315: $555-561,2004$.

This work is licensed under a Creative Commons Attribution-NonCommercial-NoDerivatives 4.0 International (CC BY-NC-ND 4.0) License. 